\title{
Asymptotic symmetry groups and operator algebras
}

\author{
Waldemar Schulgin \\ Université Libre de Bruxelles and International Solvay Institutes \\ ULB-Campus Plaine CP231 \\ B-1050 Brussels, Belgium \\ waldemar.schulgin AT ulb.ac.be
}

\author{
Jan Troost \\ Laboratoire de Physique Théoriqu@1 \\ Ecole Normale Supérieure \\ 24 rue Lhomond, 75005 Paris, France
}

\begin{abstract}
We associate vertex operators to space-time diffeomorphisms in flat space string theory, and compute their algebra, which is a diffeomorphism algebra with higher derivative corrections. As an application, we realize the asymptotic symmetry group $B M S_{3}$ of threedimensional flat space in terms of vertex operators on the string worldsheet. This provides an embedding of the $B M S_{3}$ algebra in a consistent theory of quantum gravity. Higher derivative corrections vanish asymptotically. An appendix is dedicated to $\alpha^{\prime}$ corrected algebras in conformal field theory and string theory.
\end{abstract}

\footnotetext{
${ }^{1}$ Unité Mixte du CNRS et de l'Ecole Normale Supérieure associée à l'université Pierre et Marie Curie 6 , UMR 8549.
} 


\section{Contents}

1 Introduction 3

2 The embedding in $A d S_{3}$

2.1 The embedding in $A d S_{3}$ gravity . . . . . . . . . . . . . . 4

2.2 The embedding in $A d S_{3}$ string theory $\ldots \ldots \ldots \ldots$. . . . . . . . . . . .

3 The algebra of diffeomorphism vertex operators 5

3.1 Diffeomorphism vertex operators . . . . . . . . . . . . . 5

3.2 The commutator of diffeomorphism vertex operators . . . . . . . . . . 6

3.3 Other worldsheet quantum corrections . . . . . . . . . . . . 8

3.4 Quantum corrections and marginality . . . . . . . . . . . 8

3.5 Asymptotically marginal diffeomorphisms . . . . . . . . . . . . . . 9

4 Three-dimensional flat space 9

4.1 The $B M S_{3}$ diffeomorphism vertex operators . . . . . . . . . . . . . . . 10

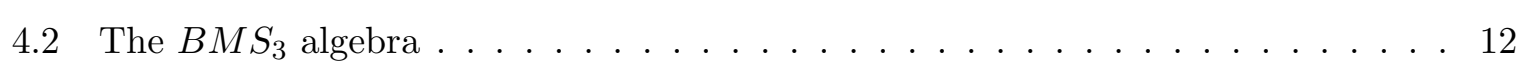

4.3 The central charge . . . . . . . . . . . . . . . . 13

5 Conclusion $\quad 14$

A Diffeomorphism operators are BRST exact 15

B Higher derivative corrections to operator algebras 16

B.1 Chiral algebras ....................... 17

B.2 A non-chiral algebra . . . . . . . . . . . . . . . . . . 21 


\section{Introduction}

Holography in flat space has an ambiguous status. On the one hand, there are arguments that quantum theories of gravity are holographic, independently of the asymptotics of the spacetime. On the other hand, concrete examples of quantum theories of gravity with a holographic dual are confined to anti-de Sitter spaces, close cousins, and low-dimensional examples. It thus remains worthwhile to investigate to what extent holography in flat space holds. If it does, one would like to construct explicit examples.

A guiding principle is the flat space asymptotic symmetry group. This group first played a role in the analysis of gravitational waves in flat space, and was later greatly elucidated [1-7]. For a review see e.g. [8] and e.g. [9] for further developments. In particular, it was shown that general relativity in three-dimensional flat space can be given boundary conditions that allow for a large $B M S_{3}$ symmetry algebra. This symmetry algebra is a contraction of two centrally extended Virasoro algebras. As such, it is a close analogue to the asymptotic symmetry group of $A d S_{3}$ [10]. The latter is suggestive of the existence of a dual conformal field theory. It thus is natural to analyze the $B M S_{3}$ algebra closely, with flat space holography in mind.

In this paper, we want to analyze to what extent the $B M S_{3}$ symmetry algebra can be embedded into a fully consistent theory of quantum gravity. We wish to show that tree level three-dimensional flat space string theory represents the asymptotic symmetry group $B M S_{3}$. We furthermore show that classes of possible $\alpha^{\prime}$ corrections to the vertex operator realization we propose are absent.

Our paper is organized as follows. In section 2, we recall how the $B M S_{3}$ algebra arises from the asymptotic symmetry algebra of $A d S_{3}$ in the limit of vanishing cosmological constant (see e.g. [11]). We discuss the differences between the case with zero and negative cosmological constant, and discuss the subtleties in taking the limit at the level of the vertex operator algebra on the worldsheet.

In section 3 we review how to associate vertex operators with diffeomorphisms in spacetime, and we compute their algebra. It turns out that there are possible $\alpha^{\prime}$ corrections to the algebra. Since this is a perhaps surprising feature of our analysis, we dedicate a long appendix to worldsheet vertex operator algebras, both chiral and non-chiral, that exhibit $\alpha^{\prime}$ corrections. We structured the appendix such that it can be read independently.

We are further lead, in section 3 to define the concept of asymptotically marginal diffeomorphisms. Indeed, we wish to remark that all diffeomorphisms leave a given background on-shell. In string theory, each diffeomorphism is associated to a BRST exact state, which therefore is BRST closed and leaves the string field on-shell. We remind the reader in appendix A how this goes in practice in covariantly quantized string theory (even when the diffeomorphisms are not transversely polarized or marginal). Still, such diffeomorphisms have a non-trivial leg in the ghost sector. That is why we will concentrate in subsection 3.5 on diffeomorphisms that only shift the metric, and that are asymptotically transverse and on-shell. They are associated to diffeomorphisms that can be written as shifts of physical matter fields of the form $c \bar{c} O$ where $O$ is a primary operator in the matter conformal field theory.

We then show in section 4 that the $B M S_{3}$ algebra can be represented by asymptotically marginal vertex operators in string theory, and moreover, that potential $\alpha^{\prime}$ corrections to the diffeomorphism algebra vanish. We conclude in section 5 with a summary and a list of topics for further analysis. 


\section{The embedding in $A d S_{3}$}

In this section, we review how the asymptotic symmetry group of three-dimensional flat space arises from that of anti-de Sitter space, and give motivation for constructing the $B M S_{3}$ algebra in flat space string theory directly.

\subsection{The embedding in $A d S_{3}$ gravity}

A first approach to the problem of constructing the $B M S_{3}$ algebra in string theory could consist in taking the limit of large radius of curvature in the $A d S_{3}$ results. Indeed, this is possible, and leads from two copies of the Virasoro algebra to the $B M S_{3}$ algebra, with central charge. We start out with two copies of the Virasoro algebra with central charge $c$ :

$$
\begin{aligned}
& {\left[\mathcal{L}_{m}, \mathcal{L}_{n}\right]=(m-n) \mathcal{L}_{m+n}+\frac{c}{12}\left(m^{3}-m\right) \delta_{m+n, 0}} \\
& {\left[\tilde{\mathcal{L}}_{m}, \tilde{\mathcal{L}}_{n}\right]=(m-n) \tilde{\mathcal{L}}_{m+n}+\frac{c}{12}\left(m^{3}-m\right) \delta_{m+n, 0} .}
\end{aligned}
$$

The classical general relativity value of the central charge is $c=\frac{3}{2} \frac{l}{G_{N}}$ where $l$ is the radius of curvature of the $A d S_{3}$ space and $G_{N}$ is Newton's constant [10. The central charge acquires $\alpha^{\prime} / l^{2}$ corrections in bosonic string theory [12,13. We can obtain the $B M S_{3}$ algebra through the redefinition

$$
\begin{aligned}
& \mathcal{P}_{m}=\frac{1}{l}\left(\mathcal{L}_{m}+\tilde{\mathcal{L}}_{-m}\right) \\
& \mathcal{J}_{m}=\mathcal{L}_{m}-\tilde{\mathcal{L}}_{-m},
\end{aligned}
$$

and the contraction $l \rightarrow \infty$ with the charges $\mathcal{P}_{m}$ and $\mathcal{J}_{m}$ kept fixed, yielding:

$$
\begin{aligned}
& {\left[\mathcal{J}_{m}, \mathcal{J}_{n}\right]=(m-n) \mathcal{J}_{m+n}} \\
& {\left[\mathcal{J}_{m}, \mathcal{P}_{n}\right]=(m-n) \mathcal{P}_{m+n}+\frac{c}{12}\left(m^{3}-m\right) \delta_{m+n, 0}} \\
& {\left[\mathcal{P}_{m}, \mathcal{P}_{n}\right]=0}
\end{aligned}
$$

where now $c=3 / G_{N}$. The limit we performed can alternatively be described as the limit of large momentum compared to the inverse radius of curvature of $A d S_{3}$. Note that the momentum charges $\mathcal{P}$ carry dimension of one over length, as does the central charge. Without reference scale (e.g. a momentum), the value of the central charge is arbitrary. Combining momentum with the three-dimensional Newton constant allows for the construction of a dimensionless ratio. We can make this manifest in the algebra:

$$
\begin{aligned}
{\left[\mathcal{J}_{m}, \mathcal{J}_{n}\right] } & =(m-n) \mathcal{J}_{m+n} \\
{\left[\mathcal{J}_{m}, G_{N} \mathcal{P}_{n}\right] } & =(m-n) G_{N} \mathcal{P}_{m+n}+\frac{1}{4}\left(m^{3}-m\right) \delta_{m+n, 0} \\
{\left[G_{N} \mathcal{P}_{m}, G_{N} \mathcal{P}_{n}\right] } & =0
\end{aligned}
$$

\subsection{The embedding in $A d S_{3}$ string theory}

The worldsheet embedding of the $A d S_{3}$ general relativity asymptotic symmetry algebra was performed in [14,15]. One can review that calculation, and take the flat space limit at the very end to recuperate the $B M S_{3}$ algebra, as we did above. That is one way to embed the 
asymptotic symmetry algebra of flat space in string theory - by viewing flat space string theory as a limit of $A d S_{3}$ string theory where we take the cosmological constant to zero.

We would like to develop a more direct route, working with strings in flat space, and the flat space worldsheet string action. One reason is the following. The calculation of [14] corresponds to an expansion in a free conformal field theory deformed by the operator $\exp (-\rho / \sqrt{k})$, where the radial coordinate $\rho$ is taken large and the radius of curvature squared over $\alpha^{\prime}$ (i.e. the level $k$ ) is kept fixed, such that one has a perturbative expansion. Taking the zero cosmological constant limit $k \rightarrow \infty$ does not commute with the large radius limit. The non-commutativity of these two limits is one way to understand that there is no direct way of adapting the calculation in 14 in $A d S_{3}$ to the flat space context proper. Furthermore, we note that the $A d S_{3}$ conformal field theory is interacting and difficult to solve while the flat space worldsheet action is a free conformal field theory. To learn about flat space string theory and its asymptotic symmetry group, we want to develop a more direct approach.

\section{The algebra of diffeomorphism vertex operators}

\subsection{Diffeomorphism vertex operators}

String theory is a theory of gravity, and is invariant under diffeomorphisms. The algebra of diffeomorphisms is part of the vast symmetry algebra of string theory. In a covariant quantization, diffeomorphisms correspond to shifts of the background by BRST exact worldsheet vertex operators. In particular, diffeomorphisms correspond to graviton vertex operators where the graviton fluctuation is taken to correspond to the variation of the metric under an infinitesimal diffeomorphism, at least to first order in $\alpha^{\prime}$. We will call such a vertex operator a (generalized) diffeomorphism vertex operator. In the classical general relativity limit of string theory, we expect the diffeomorphism vertex operators to satisfy the diffeomorphism algebra. They may satisfy an $\alpha^{\prime}$ corrected algebra at higher order, but by abuse of nomenclature, we will still call these operators diffeomorphism vertex operators.

More explicitly, there is a map from infinitesimal diffeomorphisms parameterized by a vector field $\xi$ to graviton vertex operators $V_{\xi}$ :

$$
V_{\xi}=\frac{1}{2 \pi \alpha^{\prime}} \int d^{2} z \delta G_{\mu \nu} \partial X^{\mu} \bar{\partial} X^{\nu}
$$

where the variation of the metric $\delta G_{\mu \nu}$ is:

$$
\delta G_{\mu \nu}=\nabla_{\mu} \xi_{\nu}+\nabla_{\nu} \xi_{\mu} .
$$

The first order commutator algebra of these diffeomorphism vertex operators is expected to reproduce the commutator algebra of diffeomorphisms, where the vector associated to the commutator of diffeomorphisms $\xi_{1}$ and $\xi_{2}$ is the Lie bracket $\left[\xi_{1}, \xi_{2}\right]^{\mu}=\xi_{1}^{\nu} \partial_{\nu} \xi_{2}^{\mu}-\xi_{2}^{\nu} \partial_{\nu} \xi_{1}^{\mu}$.

The structure of the algebra can be made more explicit in the case of a flat space background. In $n$-dimensional flat space, the background metric is trivial and the operator product expansions of the free coordinate fields $X^{\mu}$ are:

$$
X^{\mu}\left(z_{1}, \bar{z}_{1}\right) X^{\nu}\left(z_{2}, \bar{z}_{2}\right) \approx-\frac{\alpha^{\prime}}{2} \log \left|z_{1}-z_{2}\right|^{2} .
$$

The covariant derivatives $\nabla_{\mu}$ in equation (3.2) reduce to ordinary derivatives $\partial_{\mu}$. We can use this information to calculate the commutator of diffeomorphism vertex operators. Before 
performing the calculation, we simplify the form of the diffeomorphism vertex operators, using the worldsheet equations of motion and Stokes theorem:

$$
\begin{aligned}
V_{\xi} & =\frac{1}{2 \pi \alpha^{\prime}} \int d^{2} z\left(\partial_{\mu} \xi_{\nu}+\partial_{\nu} \xi_{\mu}\right) \partial X^{\mu} \bar{\partial} X^{\nu} \\
& =\frac{1}{2 \pi \alpha^{\prime}}\left(\oint d z \xi_{\mu} \partial X^{\mu}-\oint d \bar{z} \xi_{\mu} \bar{\partial} X^{\mu}\right) .
\end{aligned}
$$

The contour integrals are performed over boundaries of the worldsheet, and around other insertions. After these preliminaries, we are ready to compute the algebra of the (generalized) diffeomorphism vertex operators.

\subsection{The commutator of diffeomorphism vertex operators}

In string theory it is natural to extend the algebra of diffeomorphisms by the algebra of antisymmetric gauge transformations. Indeed, T-duality symmetry of string theory puts them on similar footing. We are thus motivated to define the following operators, reminiscent of current components:

$$
\begin{aligned}
j_{z}\left(\xi^{L}\right) & =\xi^{L} \cdot \partial X \\
j_{\bar{z}}\left(\xi^{R}\right) & =\xi^{R} \cdot \bar{\partial} X .
\end{aligned}
$$

where $\xi^{L, R}$ are space-time fields. We start off with an important remark on the algebra of these operators. The operators $\xi^{L}$ and $\xi^{R}$ are functionals of the coordinate fields $X^{\mu}$. We can assume they can be Fourier decomposed. The Fourier modes are exponentials with operator products:

$$
: e^{i k_{1} \cdot X\left(z_{1}, \bar{z}_{1}\right)}:: e^{i k_{2} \cdot X\left(z_{2}, \bar{z}_{2}\right)}:=\left|z_{1}-z_{2}\right|^{\alpha^{\prime} k_{1} \cdot k_{2}}: e^{i k_{1} \cdot X\left(z_{1}, \bar{z}_{1}\right)+i k_{2} \cdot X\left(z_{2}, \bar{z}_{2}\right)}: .
$$

The factor $\left|z_{1}-z_{2}\right|^{\alpha^{\prime} k_{1} \cdot k_{2}}$ changes the dimension of the product from the sum of the dimensions of the factor operators. The first important remark we want to make is that almost always we will ignore this type of contribution. That is restrictive, and we will discuss the importance of this restriction on several occasions later on. In summary, in the following we will often ignore $\xi-\xi$ contractions.

After this preliminary, we compute the algebra of diffeomorphisms and anti-symmetric gauge transformations, through the operator product expansion of the component operators. There will be both double and single contractions. The double contractions come with an extra power of $\alpha^{\prime}$ (which we set equal to two), and two extra space-time derivatives. The operator products are:

$$
\begin{aligned}
j_{z}\left(z_{1}, \bar{z}_{1}\right) j_{z}\left(z_{2}, \bar{z}_{2}\right) \approx & -\frac{1}{z_{1}-z_{2}} \xi^{L 1 \rho} \partial_{\rho} \xi_{\mu}^{2 L} \partial X^{\mu}(2)+\frac{1}{z_{1}-z_{2}} \xi^{2 L \rho} \partial_{\rho} \xi_{\mu}^{L L} \partial X^{\mu}(2) \\
& -\frac{1}{\left(z_{1}-z_{2}\right)^{2}} \xi^{L 1 \rho}(1) \xi_{\rho}^{L 2}(2)-\frac{1}{\left(z_{1}-z_{2}\right)^{2}} \partial_{\rho} \xi^{L 1 \nu}(1) \partial_{\nu} \xi^{L 2 \rho}(2)
\end{aligned}
$$

where (1) and (2) denote operators evaluated at $z_{1}$ or $z_{2}$ respectively. For the mixed operator product of current components we have:

$$
\begin{aligned}
j_{z}\left(z_{1}, \bar{z}_{1}\right) j_{\bar{z}}\left(z_{2}, \bar{z}_{2}\right) \approx & -\frac{1}{z_{1}-z_{2}} \xi^{L 1 \rho} \partial_{\rho} \xi_{\mu}^{2 R} \bar{\partial} X^{\mu}(2)+\frac{1}{\bar{z}_{1}-\bar{z}_{2}} \xi^{2 R \rho} \partial_{\rho} \xi_{\mu}^{1 L} \partial X^{\mu}(2) \\
& - \text { contact term }-\frac{1}{\left|z_{1}-z_{2}\right|^{2}} \partial_{\rho} \xi^{L 1 \nu}(1) \partial_{\nu} \xi^{R 2 \rho}(2)
\end{aligned}
$$




\section{The commutators}

We can use these operator products, valid at short distance on a cylinder as well as on the plane to compute equal time commutators of these operators. We evaluate products of operators at $z=\sigma+i \tau$, regularized by a split in the time direction $\tau$ in accord with time ordering 2 After expanding arguments around $\sigma_{1,2}$, we find:

$$
\begin{aligned}
{\left[j_{z}(1), j_{z}(2)\right]=} & 2 \pi i \delta\left(\sigma_{1}-\sigma_{2}\right)\left(-\xi^{L 1 \rho} \partial_{\rho} \xi_{\mu}^{2 L} \partial X^{\mu}+\xi^{2 L \rho} \partial_{\rho} \xi_{\mu}^{1 L} \partial X^{\mu}\right)\left(\sigma_{2}\right) \\
& +2 \pi i \partial_{\sigma_{1}} \delta\left(\sigma_{1}-\sigma_{2}\right)\left(\xi^{L 1 \rho}\left(\sigma_{1}\right) \xi_{\rho}^{L 2}\left(\sigma_{2}\right)+\partial_{\rho} \xi^{L 1 \nu}\left(\sigma_{1}\right) \partial_{\nu} \xi^{L 2 \rho}\left(\sigma_{2}\right)\right)
\end{aligned}
$$

while the mixed commutator is:

$$
\begin{aligned}
{\left[j_{z}(1), j_{\bar{z}}(2)\right]=} & 2 \pi i \delta\left(\sigma_{1}-\sigma_{2}\right)\left(-\xi^{L 1 \rho} \partial_{\rho} \xi_{\mu}^{2 R} \bar{\partial} X^{\mu}-\xi^{2 R \rho} \partial_{\rho} \xi_{\mu}^{1 L} \partial X^{\mu}\right. \\
& +\frac{1}{2}\left(\partial_{\mu} \partial_{\rho} \xi^{L 1 \nu} \partial_{\nu} \xi^{R 2 \rho} \partial X^{\mu}-\partial_{\mu} \partial_{\rho} \xi^{L 1 \nu} \partial_{\nu} \xi^{R 2 \rho} \bar{\partial} X^{\mu}\right. \\
& \left.\left.-\partial_{\rho} \xi^{L 1 \nu} \partial_{\mu} \partial_{\nu} \xi^{R 2 \rho} \partial X^{\mu}+\partial_{\rho} \xi^{L 1 \nu} \partial_{\mu} \partial_{\nu} \xi^{R 2 \rho} \bar{\partial} X^{\mu}\right)\right)\left(\sigma_{2}\right)
\end{aligned}
$$

We now recall that diffeomorphisms and anti-symmetric gauge transformations give rise to the vertex operators on the cylinder:

$$
\begin{aligned}
D[\xi] & =\frac{i}{4 \pi} \int d \sigma\left(j_{z}(\xi)-j_{\bar{z}}(\xi)\right), \\
A[\tilde{\xi}] & =\frac{i}{4 \pi} \int d \sigma\left(j_{z}(\tilde{\xi})+j_{\bar{z}}(\tilde{\xi})\right) .
\end{aligned}
$$

We obtain the commutators of the diffeomorphism charges after double integration:

$$
\begin{aligned}
{\left[D\left[\xi_{1}\right], D\left[\xi_{2}\right]\right]=} & \frac{i}{4 \pi} \int d \sigma\left(\xi^{1 \rho} \partial_{\rho} \xi_{\mu}^{2}-\xi^{2 \rho} \partial_{\rho} \xi_{\mu}^{1}\right. \\
& \left.+\frac{1}{2}\left(\partial_{\mu} \partial_{\rho} \xi^{1 \nu} \partial_{\nu} \xi^{2 \rho}-\partial_{\rho} \xi^{1 \nu} \partial_{\mu} \partial_{\nu} \xi^{2 \rho}\right)\right)\left(\partial X^{\mu}-\bar{\partial} X^{\mu}\right) .
\end{aligned}
$$

At leading order, we find the expected diffeomorphism algebra. At subleading order, we find a higher derivative contribution. We can summarize the algebra of parameters:

$$
\left[\xi_{1}, \xi_{2}\right]_{\mu}=\xi^{1 \rho} \partial_{\rho} \xi_{\mu}^{2}-\xi^{2 \rho} \partial_{\rho} \xi_{\mu}^{1}+\frac{\alpha^{\prime}}{4}\left(\partial_{\mu} \partial_{\rho} \xi^{1 \nu} \partial_{\nu} \xi^{2 \rho}-\partial_{\rho} \xi^{1 \nu} \partial_{\mu} \partial_{\nu} \xi^{2 \rho}\right)
$$

For the other brackets, we find:

$$
\begin{aligned}
& {\left[A\left[\tilde{\xi}_{1}\right], A\left[\tilde{\xi}_{2}\right]\right]=\frac{i}{4 \pi} \frac{\alpha^{\prime}}{4} \int d \sigma\left(-\partial_{\mu} \partial_{\rho} \tilde{\xi}^{1 \nu} \partial_{\nu} \tilde{\xi}^{2 \rho}+\partial_{\rho} \tilde{\xi}^{1 \nu} \partial_{\mu} \partial_{\nu} \tilde{\xi}^{2 \rho}\right)\left(\partial X^{\mu}-\bar{\partial} X^{\mu}\right)} \\
& {\left[D\left[\xi_{1}\right], A\left[\tilde{\xi}_{2}\right]\right]=\frac{i}{4 \pi} \int d \sigma\left(\xi^{1 \rho} \partial_{\rho} \tilde{\xi}_{\mu}^{2}+\partial_{\mu} \xi^{1 \rho} \tilde{\xi}_{\rho}^{2}+\frac{\alpha^{\prime}}{2} \partial_{\mu} \partial_{\rho} \xi^{1 \nu} \partial_{\nu} \tilde{\xi}^{2 \rho}\right)\left(\partial X^{\mu}+\bar{\partial} X^{\mu}\right)}
\end{aligned}
$$

We have found the algebra of pairs of parameters:

$$
\begin{aligned}
{\left[\left(\xi_{1}, \tilde{\xi}_{1}\right),\left(\xi_{2}, \tilde{\xi}_{2}\right)\right]=} & \left(\left[\xi_{1}, \xi_{2}\right], \mathcal{L}_{\xi_{1}} \tilde{\xi}_{2}-\mathcal{L}_{\xi_{2}} \tilde{\xi}_{1}-c_{0} d\left(\xi_{1} \cdot \tilde{\xi}_{2}-\xi_{2} \cdot \tilde{\xi}_{1}\right)\right) \\
& +\left(\frac{\alpha^{\prime}}{4}\left(\partial_{\mu} \partial_{\rho} \xi^{1 \nu} \partial_{\nu} \xi^{2 \rho}-\partial_{\rho} \xi^{1 \nu} \partial_{\mu} \partial_{\nu} \xi^{2 \rho}\right)-\frac{\alpha^{\prime}}{4}\left(\partial_{\mu} \partial_{\rho} \tilde{\xi}^{1 \nu} \partial_{\nu} \tilde{\xi}^{2 \rho}-\partial_{\rho} \tilde{\xi}^{1 \nu} \partial_{\mu} \partial_{\nu} \tilde{\xi}^{2 \rho}\right),\right. \\
& \left.\frac{\alpha^{\prime}}{2} \partial_{\mu} \partial_{\rho} \xi^{1 \nu} \partial_{\nu} \tilde{\xi}^{2 \rho}-\frac{\alpha^{\prime}}{2} \partial_{\mu} \partial_{\rho} \xi^{2 \nu} \partial_{\nu} \tilde{\xi}^{1 \rho}-c_{1} \frac{\alpha^{\prime}}{2} d\left(\partial_{\rho} \xi^{1 \nu} \partial_{\nu} \tilde{\xi}^{2 \rho}-\partial_{\rho} \xi^{2 \nu} \partial_{\nu} \tilde{\xi}^{1 \rho}\right)\right) .
\end{aligned}
$$

\footnotetext{
${ }^{2}$ See e.g. section 5 of $[16$ for the relevant techniques.
} 
There are coefficients $c_{0}$ and $c_{1}$ which are total derivative terms that are not fixed by our calculation. At leading order, we obtain the Courant bracket if we put $c_{0}=1 / 2$ as can be seen from the first line. (This corresponds to a mid-point prescription for the right hand side of the commutator of currents [17]. It also provides the existence of an extra automorphism for the bracket [18.) At higher order, the choice $c_{1}=1 / 2$ is equally natural. We fix these choices from here on. At subleading order, the algebra of our generalized diffeomorphism and antisymmetric gauge transformation vertex operators exhibits new features. We find $\alpha^{\prime}$ corrections to the diffeomorphism algebra. Generalized anti-symmetric gauge transformations commute into a diffeomorphism parameter. Et cetera. The algebra deserves further comment.

If we view diffeomorphisms and anti-symmetric gauge transformations as given in terms of their standard definitions in terms of manifolds and gerbes, there can be no corrections to their algebra. There is moreover every indication that they form a symmetry group of string theory at each order in the $\alpha^{\prime}$ expansion of the effective action. Here, we have mapped these classical symmetry generators to quantum vertex operators in an old covariant approach, and we have found that the algebra of operators at lowest order agrees with their geometric counterpart, while at higher order, it receives corrections. There may exist charges (which are $\alpha^{\prime}$ corrected) that will have an uncorrected geometric algebra. Nevertheless, the operators we define above are natural, and may form an alternative slice of the large symmetry algebra of string theory that could also be useful. A proper embedding in a covariant BRST framework seems primordial. These important points will turn out not to be crucial to the particular application we have in mind in this paper. In spite of this, to further frame the interesting questions that arise from these results, we give a more extensive discussion of $\alpha^{\prime}$ contributions to stringy vertex operator algebras from the point of view of two-dimensional chiral and non-chiral conformal field theory in the extended appendix $\mathrm{B}$ to this paper. In this appendix, we review the chiral first order algebra of [19], the chiral doubled algebra of [20], and how they relate to the non-chiral algebra we determined above. Further analysis of our generic result may well be fruitful.

\subsection{Other worldsheet quantum corrections}

We return to remark on a point we left aside previously. In our calculation, we dropped an overall factor of $\left|z_{1}-z_{2}\right|^{\alpha^{\prime} k_{1} \cdot k_{2}}$ in the operator product of Fourier modes with momenta $k_{1}$ and $k_{2}$. To gauge the influence of this factor on the algebra at lowest order, we suppose that $\alpha^{\prime} k_{1} \cdot k_{2}<<1$. Then, we find $\left|z_{1}-z_{2}\right|^{\alpha^{\prime} k_{1} \cdot k_{2}} \approx 1+\alpha^{\prime} k_{1} \cdot k_{2} \log \left|z_{1}-z_{2}\right|$. Therefore in the commutator we will encounter expressions of the form:

$$
\lim _{\epsilon \rightarrow 0} \frac{\log |\sigma-i \epsilon|}{\sigma-i \epsilon}-\frac{\log |\sigma+i \epsilon|}{\sigma+i \epsilon}=2 \pi i \log \sigma \delta(\sigma) .
$$

Still other contributions will be proportional to $\log \sigma \delta^{\prime}(\sigma)$. These terms are of a different type than those that give rise to the $\alpha^{\prime}$ corrections we studied above, such that it is justified to consider them separately.

\subsection{Quantum corrections and marginality}

As reviewed in appendix $\mathrm{A}$, all diffeomorphism operators in string theory, defined as the BRST operator acting on seed operators, are manifestly BRST closed. However, if we want a diffeomorphism operator to correspond strictly to a shift of a physical graviton vertex operator (proportional to the left and right ghosts $c \bar{c}$ ), then we must demand that the diffeomorphism is marginal, namely, its momentum $k$ is on-shell $\left(k^{2}=0\right)$ and transverse $(e \cdot k=0$ where $e$ 
is the polarization of the vertex operator). Let's recall how these conditions come about. For simplicity, we consider the left-moving part of a diffeomorphism charge:

$$
V_{\xi}=\frac{1}{4 \pi} \oint d z \xi_{\nu} \partial X^{\nu}
$$

or more carefully, let us consider a Fourier component:

$$
V_{e, k}=e_{\nu}(k) \partial X^{\nu} e^{i k X} .
$$

We will compute when this operator is a primary of dimension 1 on the left. To that end, we compute the operator product expansion with the left energy-momentum tensor of the theory.

$$
-\frac{1}{2} \partial X^{\mu} \partial X_{\mu}(1) \cdot V_{e, k}(2)=-i \frac{e \cdot k}{(z-w)^{3}} e^{i k X}(2)+\frac{\frac{\alpha^{\prime} k^{2}}{4}+1}{(z-w)^{2}} V_{e, k}(2)+\frac{1}{z-w} \partial V_{e, k}(2)
$$

which says that the operator $V_{e, k}$ is dimension one on the left on the condition that the polarization is transverse $(e \cdot k=0)$, and the operator is on-shell $\left(k^{2}=0\right)$.

If we consider the commutator of marginal diffeomorphism vertex operators, and moreover require that the result also be a marginal operator, then no anomalous worldsheet quantum corrections (of the type discussed in subsection 3.3) will occur. Indeed, consider two marginal diffeomorphisms, and require that they commute into a third, marginal diffeomorphism. Marginality implies that $k_{1}$ and $k_{2}$ square to zero. On the other hand, the newly generated diffeomorphism has momentum $k_{1}+k_{2}$ and will only be marginal if $\left(k_{1}+k_{2}\right)^{2}=0=2 k_{1} \cdot k_{2}$. The terms generated by contractions of the parameters of diffeomorphism among themselves will then vanish. Note that if one starts with two marginal diffeomorphisms, the result is not necessarily marginal, such that the set of marginal diffeomorphism vertex operators does not necessarily close among itself under commutation.

\subsection{Asymptotically marginal diffeomorphisms}

For our purposes however, marginality will be too strong a requirement on the diffeomorphism vertex operators we wish to consider. Rather, we will consider diffeomorphisms that we call asymptotically marginal. These are diffeomorphism operators that are marginal on the worldsheet, only up to terms which are asymptotically vanishing in space-time. This allows for sufficient freedom to realize asymptotic symmetry groups in terms of worldsheet vertex operators. These operators will act on the solution space with given asymptotic boundary conditions. We illustrate these concepts with an example in the next section.

\section{Three-dimensional flat space}

In this section, as an example of the use of the above concepts and results, we study string theory in three-dimensional flat space and its asymptotic symmetry algebra $B M S_{3}[8]$. The framework developed earlier is valid for higher dimensional flat spaces as well. We note that the $B M S_{3}$ diffeomorphisms generate the solution space in pure three-dimensional flat space gravity (as they do in $A d S_{3}$ pure gravity) [8]. Thus, these should correspond to asymptotically marginal diffeomorphisms. We will show that they do, and will show that the classes of $\alpha^{\prime}$ corrections computed previously are absent for these diffeomorphism vertex operators. In this 
section, we suppose that string theory is compactified on an appropriate space and that the non-compact directions correspond to the space-time $\mathbb{R}^{2,1}$.

The worldsheet theory for a string in (Euclidean) three-dimensional flat space is:

$$
S=\frac{1}{2 \pi \alpha^{\prime}} \int d^{2} z \partial X^{i} \bar{\partial} X_{i}
$$

where $i=1,2,3$. We will work in terms of the linearly related coordinate system of free fields $(\phi, \gamma, \bar{\gamma})$ :

$$
\begin{aligned}
X^{1} & =\phi \\
X^{2}+i X^{3} & =\gamma \\
X^{2}-i X^{3} & =\bar{\gamma} .
\end{aligned}
$$

\subsection{The $B M S_{3}$ diffeomorphism vertex operators}

We demand that the space-time be asymptotically Minkowski (see e.g. [8]). Coordinates well adapted to the calculation of the asymptotic symmetry group are $(u, r, \varphi)$ with metric:

$$
d s^{2}=-d u^{2}-2 d u d r+r^{2} d \varphi^{2} .
$$

In these coordinates, the boundary is at fixed $\varphi, u$ and radial infinity $r \rightarrow \infty$. The string worldsheet theory is interacting, and strongly interacting at large radius. A trick could consist in introducing an auxiliary coordinate to reduce the $r^{2}$ term to a $r^{-2}$ interaction, but we will not follow this route here. Rather, we will go back and forth between the asymptotic symmetry coordinates $(u, r, \varphi)$ and the free field coordinates $(\phi, \gamma, \bar{\gamma})$. The metric in the latter coordinates is

$$
d s^{2}=d \phi^{2}+d \gamma d \bar{\gamma},
$$

and the mapping between the coordinate sets is

$$
\begin{aligned}
\phi & =-i(u+r) \\
\gamma & =r e^{i \varphi} \\
\bar{\gamma} & =r e^{-i \varphi} .
\end{aligned}
$$

The components of the vector fields representing the infinitesimal diffeomorphisms that generate the $B M S_{3}$ algebra are [8] :

$$
\begin{aligned}
& \xi^{u}=T+u Y^{\prime} \\
& \xi^{\varphi}=Y-\frac{1}{r}\left(T^{\prime}+u Y^{\prime \prime}\right) \\
& \xi^{r}=-r Y^{\prime}+T^{\prime \prime}+u Y^{\prime \prime \prime},
\end{aligned}
$$

where the functions $T$ and $Y$ are functions of the angular coordinate $\varphi$ only. Primes on the functions $Y$ and $T$ will correspond to the derivatives with respect to the $\varphi$ coordinate.

We wish to write down the vertex operators corresponding to the $B M S_{3}$ diffeomorphisms in the free field theory variables. To do this, we perform the coordinate transformation to the $(\phi, \gamma, \bar{\gamma})$ variables on the vector fields. Next, we Fourier decompose the generators $T$ and $Y$ in $\varphi$. With those results, we can compute the corresponding vertex operators, in free field variables. The results are as follows. 


\subsubsection{The translation vertex operators}

We find the components of the translation diffeomorphisms corresponding to the $n$-th Fourier mode of $T$ :

$$
\begin{aligned}
& \xi_{T, n}^{\phi}=-i\left(1-n^{2}\right)\left(\frac{\gamma}{\bar{\gamma}}\right)^{\frac{n}{2}}, \\
& \xi_{T, n}^{\gamma}=-n(n-1)\left(\frac{\gamma}{\bar{\gamma}}\right)^{\frac{n+1}{2}}, \\
& \xi_{T, n}^{\bar{\gamma}}=-n(n+1)\left(\frac{\gamma}{\bar{\gamma}}\right)^{\frac{n-1}{2}} .
\end{aligned}
$$

The corresponding vertex operators $V_{T, n}$ are:

$$
\begin{aligned}
V_{T, n}= & \frac{1}{8 \pi} \oint d z\left(-2 i\left(1-n^{2}\right)\left(\frac{\gamma}{\bar{\gamma}}\right)^{\frac{n}{2}} \partial \phi-n(n+1)\left(\frac{\gamma}{\bar{\gamma}}\right)^{\frac{n-1}{2}} \partial \gamma-n(n-1)\left(\frac{\gamma}{\bar{\gamma}}\right)^{\frac{n+1}{2}} \partial \bar{\gamma}\right) \\
& -\frac{1}{8 \pi} \oint d \bar{z}\left(-2 i\left(1-n^{2}\right)\left(\frac{\gamma}{\bar{\gamma}}\right)^{\frac{n}{2}} \bar{\partial} \phi-n(n+1)\left(\frac{\gamma}{\bar{\gamma}}\right)^{\frac{n-1}{2}} \bar{\partial} \gamma-n(n-1)\left(\frac{\gamma}{\bar{\gamma}}\right)^{\frac{n+1}{2}} \bar{\partial} \bar{\gamma}\right) .
\end{aligned}
$$

\subsubsection{The rotation vertex operators}

For the Fourier modes of infinitesimal rotation diffeomorphisms, we find:

$$
\begin{aligned}
& \xi_{Y, n}^{\phi}=n\left(i\left(1-n^{2}\right) \phi+\left(n^{2}-2\right) \sqrt{\gamma \bar{\gamma}}\right)\left(\frac{\gamma}{\bar{\gamma}}\right)^{\frac{n}{2}} \\
& \xi_{Y, n}^{\gamma}=(n-1)\left(n^{2} \phi+i\left(n^{2}-1\right) \sqrt{\gamma \bar{\gamma}}\right)\left(\frac{\gamma}{\bar{\gamma}}\right)^{\frac{n+1}{2}} \\
& \xi_{Y, n}^{\bar{\gamma}}=(n+1)\left(n^{2} \phi+i\left(n^{2}-1\right) \sqrt{\gamma \bar{\gamma}}\right)\left(\frac{\gamma}{\bar{\gamma}}\right)^{\frac{n-1}{2}} .
\end{aligned}
$$

These components lead to the vertex operators $V_{Y, n}$ :

$$
\begin{aligned}
V_{Y, n}=\frac{1}{8 \pi} \oint d z\left(2 n\left(i\left(1-n^{2}\right) \phi+\left(n^{2}-2\right) \sqrt{\gamma \bar{\gamma}}\right)\left(\frac{\gamma}{\bar{\gamma}}\right)^{\frac{n}{2}} \partial \phi\right. \\
+(n+1)\left(n^{2} \phi+i\left(n^{2}-1\right) \sqrt{\gamma \bar{\gamma}}\right)\left(\frac{\gamma}{\bar{\gamma}}\right)^{\frac{n-1}{2}} \partial \gamma \\
\left.+(n-1)\left(n^{2} \phi+i\left(n^{2}-1\right) \sqrt{\gamma \bar{\gamma}}\right)\left(\frac{\gamma}{\bar{\gamma}}\right)^{\frac{n+1}{2}} \partial \bar{\gamma}\right) \\
-\frac{1}{8 \pi} \oint d \bar{z}\left(2 n\left(i\left(1-n^{2}\right) \phi+\left(n^{2}-2\right) \sqrt{\gamma \bar{\gamma}}\right)\left(\frac{\gamma}{\bar{\gamma}}\right)^{\frac{n}{2}} \bar{\partial} \phi\right. \\
+(n+1)\left(n^{2} \phi+i\left(n^{2}-1\right) \sqrt{\gamma \bar{\gamma}}\right)\left(\frac{\gamma}{\bar{\gamma}}\right)^{\frac{n-1}{2}} \bar{\partial} \gamma \\
\left.+(n-1)\left(n^{2} \phi+i\left(n^{2}-1\right) \sqrt{\gamma \bar{\gamma}}\right)\left(\frac{\gamma}{\bar{\gamma}}\right)^{\frac{n+1}{2}} \bar{\partial} \bar{\gamma}\right) .
\end{aligned}
$$




\subsubsection{Asymptotic marginality}

We now wish to check whether the $B M S_{3}$ diffeomorphism vertex operators satisfy the conditions of asymptotic marginality. We perform this calculation in the free field flat space variables $(\phi, \gamma, \bar{\gamma})$ in which we derived the conditions of transversality:

$$
\partial_{\mu} \xi^{\mu}=0
$$

and masslessness:

$$
\partial_{\mu} \partial^{\mu} \xi^{\nu}=0
$$

After a calculation, we find that transversality is satisfied exactly for the translation as well as the rotation charges. The calculation involves non-trivial cancellations between the coefficients, dependent on the Fourier momentum $n$. We moreover have that the massless, or marginality condition is satisfied for the translation charges up to terms that go like $r^{-2}$ and for the rotation charges up to terms that go like $r^{-1}$. This comes about because the non-trivial contributions arise from $\gamma$ and $\bar{\gamma}$ derivatives, which lower the power of the radial coordinate $r$ in the diffeomorphism parameter by 2. Taking into account the leading term in the parameter (which is $O\left(r^{0}\right)$ for translations and $O(r)$ for rotations), we find the quoted suppression factors. From appendix $\mathrm{A}$ it should be clear that we are comparing the momentum squared contribution in the worldsheet conformal dimension to the leading contribution of 1 arising from a worldsheet derivative in the diffeomorphism vertex operator. Hence, at large radius (compared to the string length), these terms indeed are negligible. Thus, the $B M S_{3}$ diffeomorphisms are asymptotically marginal.

\subsection{The $B M S_{3}$ algebra}

We have already established that to leading order in $\alpha^{\prime}$ the (generalized, asymptotically marginal) diffeomorphism vertex operators that we constructed satisfy the algebra of ordinary diffeomorphisms. Thus, for the $B M S_{3}$ diffeomorphisms, they satisfy the $B M S_{3}$ algebra. We have proven this by going to a coordinate system where the worldsheet fields are free, then performing the operator products, and commutators, after which we return to the coordinate system handy in the definition and analysis of the asymptotics. Thus, the embedding of the $B M S_{3}$ algebra in a consistent theory of quantum gravity has been obtained. In this subsection, we show that the potential $\alpha^{\prime}$ corrections we computed are subleading asymptotically, and that therefore the $B M S_{3}$ algebra is represented without $\alpha^{\prime}$ corrections.

To establish these facts, we first distinguish three types of commutators, namely between translations, between rotations and translations, and between rotations. Firstly, as an illustrative example, we present the computation of the higher derivative corrections to the algebra of rotational diffeomorphisms. We recall the algebra we derived in (3.12). We must be careful to apply the formula to diffeomorphisms expressed in the free field coordinates $(\phi, \gamma, \bar{\gamma})$. Afterwards, we express the coefficients in the coordinate system $(r, u, \varphi)$ to simplify the task of 
comparing orders of coefficients in the limit of fixed $u, \varphi$ and $r \rightarrow \infty$. In practice, we find:

$$
\begin{aligned}
{\left[\xi_{Y, n}, \xi_{Y, m}\right]^{\phi}=} & -i(n-m) \xi_{Y, n+m}^{\phi}+\frac{i m^{2} n^{2}\left(n^{2}-m^{2}\right) u^{2} e^{i(m+n) \varphi}}{r} \\
& +\frac{\alpha^{\prime}}{4} \frac{i\left(n^{2}-m^{2}\right)\left(n^{2}+m^{2}-1\right) e^{i(n+m) \varphi}}{r}+\mathcal{O}\left(\frac{1}{r^{2}}\right), \\
{\left[\xi_{Y, n}, \xi_{Y, m}\right]^{\gamma}=} & -i(n-m) \xi_{Y, n+m}^{\gamma}-\frac{m^{2} n^{2}\left(n^{2}-m^{2}\right) u^{2} e^{i(m+n+1) \varphi}}{r} \\
& +\frac{\alpha^{\prime}}{4} \frac{(m-n)(m+n-1)\left(m^{2}+n^{2}-m-n-2\right) e^{i(n+m-1) \varphi}}{2 r}+\mathcal{O}\left(\frac{1}{r^{2}}\right), \\
{\left[\xi_{Y, n}, \xi_{Y, m}\right]^{\bar{\gamma}}=} & -i(n-m) \xi_{Y, n+m}^{\bar{\gamma}}-\frac{m^{2} n^{2}\left(n^{2}-m^{2}\right) u^{2} e^{i(m+n-1) \varphi}}{r} \\
& +\frac{\alpha^{\prime}}{4} \frac{(m-n)(m+n+1)\left(m^{2}+n^{2}+m+n-2\right) e^{i(n+m+1) \varphi}}{2 r}+\mathcal{O}\left(\frac{1}{r^{2}}\right) .
\end{aligned}
$$

All corrections are subleading with respect to the asymptotic algebra of diffeomorphisms. Indeed, comparing to the $\phi, \gamma, \bar{\gamma}$-components of the rotational diffeomorphisms expressed in $r, u, \varphi$ coordinates:

$$
\begin{aligned}
\xi_{Y, n}^{\phi} & =-n\left(r+\left(n^{2}-1\right) u\right) e^{i n \varphi} \\
\xi_{Y, n}^{\gamma} & =-i(n-1)\left(r+n^{2} u\right) e^{i(n+1) \varphi} \\
\xi_{Y, n}^{\bar{\gamma}} & =-i(n+1)\left(r+n^{2} u\right) e^{i(n-1) \varphi},
\end{aligned}
$$

we see that the corrections in equation (4.12) are subleading in the asymptotic region at fixed Fourier momentum. Also, we find that the $\alpha^{\prime}$ corrections of the form $\partial_{\mu} \partial_{\rho} \xi^{\nu} \partial_{\nu} \xi^{\rho}$ are entirely absent for translations, while for rotations acting on translations, we need to compare the corrections to the translation diffeomorphisms:

$$
\begin{aligned}
& \xi_{T, n}^{\phi}=-i\left(1-n^{2}\right) e^{i n \varphi}, \\
& \xi_{T, n}^{\gamma}=-n(n-1) e^{i(n+1) \varphi}, \\
& \xi_{T, n}^{\bar{\gamma}}=-n(n+1) e^{i(n-1) \varphi} .
\end{aligned}
$$

We find that the $\alpha^{\prime}$ corrections are down by a power of $r$ with respect to these coefficients. We thus find that the asymptotic symmetry algebra is well represented by our asymptotically marginal vertex operators, even when including $\alpha^{\prime}$ corrections.

Moreover, we note that the condition of asymptotic marginality satisfied by $\xi_{1}, \xi_{2}$ and their commutator, implies that the corrections proportional to $\alpha^{\prime} k_{1} \cdot k_{2}$ are also absent. Alternatively, this follows from an argument similar to the one given in subsection 4.1.3 that guaranteed asymptotic marginality.

\subsection{The central charge}

Let's consider in more detail commutators that give rise to a central charge contribution. These are commutators of the form:

$$
\left[\mathcal{J}_{m}, \mathcal{P}_{-m}\right]=2 m \mathcal{P}_{0}+\frac{c}{12}\left(m^{3}-m\right)
$$


Thus, to see the central charge, we need to match up Fourier momenta. When Fourier momenta match, we expect terms in the commutator which are formally total derivatives. However, we may generate operators like $\partial_{\sigma} \varphi$, which can give non-zero contributions to the integral for a worldsheet profile with winding number $w$ around the $\varphi$ circle. The central charge will jump when one crosses such a domain wall macroscopic string. This bears similarities to what happens in $A d S_{3}$ [14. Let's see how this manifests in a string theory context.

In $A d S_{3}$ solutions to string theory that arise from near-brane limits of the F1-NS5 system, the central charge is given by $c=6 N_{1} N_{5}$. Changing the number of fundamental strings $N_{1}$ by $\Delta N_{1}$ changes the central charge by $\Delta c=6 \Delta N_{1} N_{5}$ [14. We can think of our background as arising from considering a large number $N_{5}$ of NS5-branes, such that the radius of curvature $R=\sqrt{N_{5} \alpha^{\prime}}$ becomes large with respect to the string scale. One over the three-dimensional Newton constant, meanwhile, is proportional to $N_{1} \sqrt{N_{5}}$. It is fixed by an attractor mechanism. Changing the number of fundamental strings considered in the near-brane limit corresponds then to a jump in the three-dimensional Newton constant. It is this change in the central charge that we detect in the worldsheet calculation. The normalization factor will be:

$$
\frac{1}{G_{N}}=4 \frac{\sqrt{N_{5}} N_{1}}{\sqrt{\alpha^{\prime}}}
$$

such that the jump in the inverse Newton constant when we cross a macroscopic fundamental string will be $4 \sqrt{N_{5}} \Delta N_{1} / \sqrt{\alpha^{\prime}}$ where $\Delta N_{1}=w$ will correspond to the number of times the macroscopic worldsheet wraps the angular direction $\varphi$.

For the evaluation of the central charge in the $\mathbb{R}^{2,1}$ vacuum, a more elaborate analysis is necessary. It was performed in the $A d S_{3}$ case in [13. The analogue of this calculation in $\mathbb{R}^{2,1}$, as well as fleshing out our intuitive description of the change in central charge when crossing a fundamental string, we leave for future work.

\section{Conclusion}

We analyzed $\alpha^{\prime}$ corrections to vertex operator algebras in string theory, and to operator algebras in two-dimensional conformal field theory. We applied our analysis of possible $\alpha^{\prime}$ corrections to the $B M S_{3}$ algebra, the asymptotic symmetry algebra of three-dimensional flat space. We showed that the algebra can be represented in string theory, a consistent theory of quantum gravity, and that potential higher derivative corrections are absent. Many open research directions have become more concrete. We name a few:

- Can one use an analogue of bulk-boundary propagators to construct an exact version of the $B M S_{3}$ algebra, valid everywhere in the bulk (as in the case of $A d S_{3}$ [15])?

- Give an interpretation of the representation theory of the $B M S_{3}$ algebra in terms of the bulk gravitational theory.

- Apply our formalism to other asymptotic symmetry algebras. The application to $B M S_{4}$, or four-dimensional Minkowski space is straightforward while curved bulk spaces require more work on worldsheet vertex operator algebras.

- Interpret the Jacobiators of $\alpha^{\prime}$ corrected non-chiral vertex operator algebras, and their space-time counterparts in an algebraic and in a geometric framework.

- Embed our analysis in closed string field theory. 
- Analyze whether effective actions satisfy a constraint because of an $\alpha^{\prime}$ corrected gauge algebra.

- Extend/restrict our analysis to electromagnetism in three-dimensional flat space. (See also 21.)

- Extend the algebra to include supersymmetry.

Our work is but a step towards an improved understanding of how the work on asymptotic symmetry groups in gravity is embedded in string theory. One may legitimately hope that studying the symmetry of quantum theories of gravity will further our understanding of holography.

\section{Acknowledgments}

We would like to thank Costas Bachas, Glenn Barnich, Marc-Thierry Jaekel, Amir-Kian KashaniPoor, Pierre-Henry Lambert and Giuseppe Policastro for interesting discussions. The work of W.S. is partially supported by a Marina Solvay fellowship, by IISN - Belgium (conventions 4.4511.06 and 4.4514.08), by the "Communauté Française de Belgique" through the ARC program and by the ERC through the "SyDuGraM" Advanced Grant. This work was supported in part by the ANR grant ANR-09-BLAN-0157-02.

\section{A Diffeomorphism operators are BRST exact}

In this appendix we discuss an elementary aspect of diffeomorphism vertex operators. Diffeomorphism vertex operators arise from the action of the BRST operator $Q_{B}$ on a seed vertex operator $S$. As such, they will be BRST closed, independent of the chosen seed $S$. In particular, there will be no further constraint necessary in order for them to be on-shell in that sense. We wish to demonstrate this elementary fact explicitly.

We will concentrate on the left-movers only, and start out with a seed vertex operator $S$ which is an exponential with momentum $k$ :

$$
S=: e^{i k X}:
$$

in a theory of free scalar fields $X$, i.e. flat space string theory. Next, we compute the commutator with the BRST charge $Q_{B}$, which is given as an integral over the BRST current $j_{B}$ (see e.g. [22]):

$$
\begin{aligned}
j_{B} & =c T^{\text {matter }}+: b c \partial c:+\frac{3}{2} \partial^{2} c \\
Q_{B} & =\frac{1}{2 \pi i} \oint d z j_{B} .
\end{aligned}
$$

The commutator gives the vertex operator $V$ :

$$
V=\left[Q_{B}, S(w)\right]=\oint_{C_{w}} \frac{d z}{2 \pi i} j_{B}(z) S(w)=c \partial S(w)+\frac{\alpha^{\prime} k^{2}}{4} \partial c S(w) .
$$

as follows from the operator product expansion:

$$
T^{\text {matter }}(z) S(w) \sim \frac{\frac{\alpha^{\prime} k^{2}}{4}}{(z-w)^{2}} S(w)+\frac{1}{z-w} \partial S(w)+\ldots
$$


To confirm that this is a BRST exact vertex operator, we continue the analysis and compute the commutator of the BRST operator $Q_{B}$ and the vertex operator $V$, which is based on the operator product expansion of the energy momentum tensor with the field $\partial S$ and the OPE of $b c \partial c$ with the ghost $c$ and its derivative $\partial c$ :

$$
\begin{aligned}
T^{\text {matter }}(z) \partial S(w) & \sim \frac{\frac{\alpha^{\prime} k^{2}}{2}}{(z-w)^{3}} S(w)+\frac{\frac{\alpha^{\prime} k^{2}}{4}+1}{(z-w)^{2}} \partial S(w)+\frac{1}{z-w} \partial^{2} S(w)+\ldots \\
b c \partial c(z) c(w) & \sim \frac{1}{z-w} c \partial c(w)+\ldots \\
b c \partial c(z) \partial c(w) & \sim \frac{1}{(z-w)^{2}} c \partial c(w)+\frac{1}{z-w} c \partial^{2} c(w)+\ldots
\end{aligned}
$$

The field $\partial S$ is a quasi-primary field. For the commutator we find:

$$
\begin{aligned}
{\left[Q_{B}, V(w)\right]=} & \partial^{2} c c \frac{\alpha^{\prime} k^{2}}{4} S(w)+\partial c c\left(\frac{\alpha^{\prime} k^{2}}{4}+1\right) \partial S(w)+\frac{\alpha^{\prime} k^{2}}{4} c \partial c \partial S(w) \\
& +c \partial c \partial S(w)+\frac{\alpha^{\prime} k^{2}}{4} c \partial^{2} c S(w) \\
= & 0 .
\end{aligned}
$$

From this exercise we see that there will be no constraints on the momentum (or polarization) of a diffeomorphism vertex operator for it to be BRST closed. In that sense, it is always on-shell.

We also see from (an easy extension of) the above calculation that if we wish the diffeomorphism vertex operator to correspond to a shift only of the physical graviton vertex operator proportional to $c \bar{c} O$ where $O$ is a matter primary of dimensions $(1,1)$, then we must demand that the seed operator $S$ is massless and that the diffeomorphism is transverse. Such a diffeomorphism vertex operator, we call marginal.

\section{B Higher derivative corrections to operator algebras}

In this appendix, we analyze algebras of currents and charges in chiral and non-chiral conformal field theory, and their higher derivative corrections. Abstract algebras in two-dimensional conformal field theories have been useful in finding exact solutions to spectral problems as well as correlation functions. They have very interesting connections with various branches of mathematics, including affine Kac-Moody algebras, generalized geometry and deformation theory. They also serve as basic building blocks for symmetries in string theory, including isometries of target space, T-dualities, asymptotic symmetry groups as well as gauge symmetries like diffeomorphisms. It is the latter application we have in mind in the bulk of the paper.

In particular, we compute a number of these worldsheet algebras, and their Jacobiator. Firstly, we remark that the Jacobiator of chiral current algebras is a total derivative by the theory of vertex operator algebras [23]. Next, we compute the Jacobiator for a conformal field theory algebra with $\alpha^{\prime}$ corrections associated to a first order formalism for worldsheet sigmamodels [19], and for an example based on the chiral algebra of a free scalar field 3 These chiral algebras are warm-up examples for the non-chiral algebra we study next, of diffeomorphism and anti-symmetric gauge transformation operators. In particular, we analyze non-chiral current algebras that at leading order form a Courant algebra, and compute higher order corrections. We also calculate the ensuing Jacobiator which contains total derivative terms, and extra terms.

\footnotetext{
${ }^{3}$ This algebra was independently calculated in [20] where the Jacobiator was computed as well. Moreover, 20] developed a theory of invariant tensors with applications to $\alpha^{\prime}$ corrected doubled geometry.
} 


\section{B.1 Chiral algebras}

In this first subsection, we concentrate on chiral algebras, associated to (holomorphic) vertex operator algebras. When we analyze algebras of charges based on contour integrals of holomorphic vertex operators, we can make good use of the mathematics of vertex operator algebras (see e.g. 23] for a very readable account). We can use the general theory to argue for the fact that Jacobiators are total derivatives, and that the algebra of integrated charges satisfies the Jacobi identity. In this section, we recall a few facts of the general theory of vertex operators algebras and apply it to two algebras of charges. One example is related to $\beta \gamma$ systems [19], and another is based on the vertex operator algebra of a chiral boson and was also discussed in 20] recently.

\section{B.1.1 The general theory}

Vertex operator algebras contain a multitude of algebraic structures. One is the $(n)$-product of vertex operators which to vertex operators $a$ and $b$ associates the residue of the $(n+1)$ st pole in the operator product of mutually local vertex operators $a(z)$ and $b(w)$, expanded at $w$. That leads to the formula for mutually local operators:

$$
a(z) b(w)=\sum_{j=0}^{N-1} \frac{a(w)_{(j)} b(w)}{(z-w)^{j+1}}+: a(z) b(w):
$$

We note that the zeroth product coincides with a contour integral action:

$$
a(w)_{(0)} b(w)=\oint_{w} d z a(z) b(w),
$$

which in turn is equivalent to a commutator. The associativity of the product of vertex operators evaluated at different points implies a large number of properties of the $(n)$-products. Using these properties, the Jacobiator of the (0)-product can be computed, and it is guaranteed to be a total derivative. Explicitly, it is given by:

$$
\begin{gathered}
a(w)_{(0)}\left(b(w)_{(0)} c(w)\right)+c_{(0)}\left(a(w)_{(0)} b(w)\right)+b(w)_{(0)}\left(c(w)_{(0)} a(w)\right)= \\
-\sum_{j=1}^{\infty}(-1)^{j} \partial_{w}^{(j)}\left(c(w)_{(j)}\left(a(w)_{(0)} b(w)\right)+b(w)_{(0)}\left(c(w)_{(j)} a(w)\right)\right) .
\end{gathered}
$$

This is a standard result in the sense that it is known that modulo the derivative of the vertex operator algebra, the Jacobi identity is satisfied. Less well known seems to be the fact that the Jacobiator can be calculated in terms of the $(n)$-products of the operators, and that this identity is part of the structure of a strongly homotopy Lie algebra [24]. Strongly homotopy Lie algebras are known to arise in covariant string field theory [25, 26]. It is gratifying to see them feature in the elementary context of chiral conformal field theory as well.

In the following, we give two examples of chiral vertex operator algebras in which the zeroth product gives rise to an interesting bracket operation on a set of vertex operators. Using the general structure of vertex operator algebras, we will be guaranteed a total derivative Jacobiator, which we compute. We are particularly interested in bracket operations that contain higher derivative $\left(\alpha^{\prime}\right)$ corrections. 


\section{B.1.2 An algebra in first order formalism}

A first interesting chiral algebra that contains $\alpha^{\prime}$ corrections was discussed in [19]. It uses holomorphic vertex operators only, and contour manipulations. We define two chiral vertex operators $p$ and $X$ which satisfy the following operator products:

$$
p_{\mu}(z) X^{\nu}(w) \sim-\frac{\delta_{\mu}^{\nu}}{z-w} .
$$

We can think of the algebra as arising from a first order action principle for a free chiral boson. We now wish to analyze an algebra of charges and vertex operators given by the following expressions:

$$
\begin{aligned}
& r(f, k)=\frac{1}{2 \pi i} \oint d z f_{\mu} e^{i k_{\nu} X^{\nu}} \partial X^{\mu}, \\
& n(e, k)=\frac{1}{2 \pi i} \oint d z e^{\mu} e^{i k_{\nu} X^{\nu}} p_{\mu} .
\end{aligned}
$$

The relevant operator product expansion for the factors appearing in these charges are:

$$
p_{\mu}(z) e^{i k X(w)} \sim-\frac{i k_{\mu}}{z-w} e^{i k \cdot X(w)} .
$$

We will not only study the algebra of charges $r, n$ but also the vertex operator algebra of their integrands, $R=f_{\mu} e^{i k_{\nu} X^{\nu}} \partial X^{\mu}$ and $N=e^{\mu} e^{i k_{\nu} X^{\nu}} p_{\mu}$. In particular, let's consider the operator product of two operators $N$ :

$$
\begin{aligned}
N_{1}\left(z_{1}\right) N_{2}\left(z_{2}\right) \sim & \frac{1}{\left(z_{1}-z_{2}\right)^{2}} e_{1} \cdot k_{2} e_{2} \cdot k_{1} e^{i\left(k_{1}+k_{2}\right) \cdot X\left(z_{2}\right)} \\
& -\frac{i}{z_{1}-z_{2}}\left(e_{1} \cdot k_{2} e_{2} \cdot p-e_{2} \cdot k^{1} e_{1} \cdot p\right) e^{i\left(k_{1}+k_{2}\right) \cdot X}\left(z_{2}\right) \\
& +\frac{i}{z_{1}-z_{2}} e_{1} \cdot k_{2} e_{2} \cdot k_{1} k_{1} \cdot \partial X e^{i\left(k_{1}+k_{2}\right) \cdot X}\left(z_{2}\right) \\
& + \text { regular }
\end{aligned}
$$

In the language of the $(n)$-products, we can reformulate the operator product expansion as:

$$
\begin{aligned}
N_{1}\left(z_{2}\right)_{(1)} N_{2}\left(z_{2}\right)= & e_{1} \cdot k_{2} e_{2} \cdot k_{1} e^{i\left(k_{1}+k_{2}\right) X\left(z_{2}\right)} \\
N_{1}\left(z_{2}\right)_{(0)} N_{2}\left(z_{2}\right)= & -i\left(e_{1} \cdot k_{2} e_{2} \cdot p-e_{2} \cdot k_{1} e_{1} \cdot p\right) e^{i\left(k_{1}+k_{2}\right) X}\left(z_{2}\right) \\
& +i e_{1} \cdot k_{2} e_{2} \cdot k_{1} k_{1} \cdot \partial X e^{i\left(k_{1}+k_{2}\right) X}\left(z_{2}\right)
\end{aligned}
$$

We note that for holomorphic vertex operators, we have that the commutator of their contour integrals is given by the contour integral of the (0)-product. Thus, the properties of the (0)product of integrands will largely determine the properties of the charges (i.e. the integrated vertex operators). The algebra of the vertex operators that appear as integrands in our charges is:

$$
R_{1(0)} R_{2}=0 \quad R_{(0)} N=i e \cdot k_{1} f \cdot \partial X e^{i\left(k_{1}+k_{2}\right) X}-i e \cdot f k_{1} \cdot \partial X e^{i\left(k_{1}+k_{2}\right) X} .
$$

After Fourier transformation, we can define the operators $n$ and $r$ as [19]:

$$
n=\frac{1}{2 \pi i} \oint d z v^{\mu} p_{\mu} \quad r=\frac{1}{2 \pi i} \oint d z \omega_{\mu} \partial X^{\mu},
$$


and find the non-zero commutators:

$$
\begin{gathered}
{\left[n_{v_{1}}, n_{v_{2}}\right]=n_{\left[v_{2}, v_{1}\right]}+r_{\Omega\left(v_{1}, v_{2}\right)} \quad\left[r_{\omega}, n_{v}\right]=r_{\mathcal{L}_{v} \omega}} \\
\Omega_{\mu}\left(v_{1}, v_{2}\right)=-\frac{1}{2}\left(\partial_{\mu} \partial_{\nu} v_{1}^{\rho} \partial_{\rho} v_{2}^{\nu}-\partial_{\mu} \partial_{\nu} v_{2}^{\rho} \partial_{\rho} v_{1}^{\nu}\right),
\end{gathered}
$$

where $\omega$ is considered a one-form and $v$ a vector. Note that this agrees with the algebra of [19].4 We used that we can neglect total derivatives in the parameter of the $r$-charge. The algebra contains a higher derivative $\alpha^{\prime}$ correction. This is a basic example of the type of correction we wish to analyze.

\section{The Jacobiator}

The only non-trivial Jacobiator is the one where we consider three $n$-operators. Using the $(j)$ product formalism, we see that the Jacobiator contains terms with (1)-products at most. We thus have for the Jacobiator $\left[n_{1},\left[n_{2}, n_{3}\right]\right]+$ cyclic $=r_{\omega}$ where $\omega$ is a total derivative determined by (see formula B.3):

$$
\begin{aligned}
& \partial_{w}\left(V_{3}(w)_{(1)}\left(V_{1}(w)_{(0)} V_{2}(w)\right)+V_{2}(w)_{(0)}\left(V_{3}(w)_{(1)} V_{1}(w)\right)\right) \\
= & \partial_{w}\left(V_{3}(w)_{(1)}\left(-i\left(e_{1} \cdot k_{2} e_{2} \cdot p-e_{2} \cdot k_{1} e_{1} \cdot p\right) e^{\left.i k_{1}+k_{2}\right) X}(w)\right)\right. \\
& \left.+V_{2}(w)_{(0)}\left(e_{3} \cdot k_{1} e_{1} \cdot k_{3} e^{i\left(k_{1}+k_{3}\right) X(w)}\right)\right) \\
= & \left(-i e_{1} \cdot k_{2} e_{2} \cdot k_{3} e_{3} \cdot k_{1}+i e_{1} \cdot k_{3} e_{2} \cdot k_{1} e_{3} \cdot k_{2}-i e_{1} \cdot k_{2} e_{2} \cdot k_{3} e_{3} \cdot k_{2}\right. \\
& \left.-i e_{1} \cdot k_{3} e_{2} \cdot k_{3} e_{3} \cdot k_{1}-i e_{1} \cdot k_{2} e_{2} \cdot k_{1} e_{3} \cdot k_{1}\right) \partial_{w} e^{i\left(k_{1}+k_{2}+k_{3}\right) X(w)} .
\end{aligned}
$$

We conclude that in the vertex operator formalism, the Jacobiator has total derivative parameter $\omega$ given by 5 :

$$
\begin{aligned}
\omega_{\mu} & =\partial_{\mu} S_{1 s t} \\
S_{1 s t} & =\frac{1}{3} \partial_{\nu} \xi_{[1}^{\rho} \partial_{\rho} \xi_{2}^{\sigma} \partial_{\sigma} \xi_{3]}^{\nu}+\left(\xi_{1}^{\rho} \partial_{\rho} \partial_{\nu} \xi_{2}^{\sigma} \partial_{\sigma} \xi_{3}^{\nu}+\text { cycl. perm }\right)
\end{aligned}
$$

It is crucial to remark that the total derivative Jacobiator depends on the choice of total derivative terms in the commutator. To reproduce the above Jacobiator from the brackets, one would use the choice of total derivative terms:

$\left[n_{v_{1}}, n_{v_{2}}\right]=n_{\left[v_{2}, v_{1}\right]}+r_{\Omega\left(v_{1}, v_{2}\right)} \quad\left[n_{v}, r_{\omega}\right]=-r_{v^{\rho} \partial_{\rho} \omega_{\mu}+\partial_{\mu} v^{\rho} \omega_{\rho}} \quad \Omega_{\mu}\left(v_{1}, v_{2}\right)=-\partial_{\mu} \partial_{\nu} v_{1}^{\rho} \partial_{\rho} v_{2}^{\nu}$.

This choice is dictated by the fact that the $\mu$ derivative, arising from the Taylor expansion of an operator, by convention in vertex operator algebras, is always performed on the first operator, and therefore acts on the first parameter in the commutator brackets. We can simplify the Jacobiator by making a particular choice of brackets. Several choices give a simple Jacobiator result. One choice is the original one we made in equation (B.10), while another choice is:

$$
\left[n_{v_{1}}, n_{v_{2}}\right]=n_{\left[v_{2}, v_{1}\right]}+r_{\Omega\left(v_{1}, v_{2}\right)} \quad\left[n_{v}, r_{\omega}\right]=-r_{v^{\rho} \partial_{\rho} \omega_{\mu}-\partial_{\mu} \omega_{\rho} v^{\rho}} \quad \Omega_{\mu}\left(v_{1}, v_{2}\right)=-\partial_{\mu} \partial_{\nu} v_{1}^{\rho} \partial_{\rho} v_{2}^{\nu} .
$$

\footnotetext{
${ }^{4}$ Up to a minor typo in [19, and a different convention for $\alpha^{\prime}$.

${ }^{5}$ Our convention for anti-symmetrization is $[a b]=a b-b a$.
} 
In both cases the seed function is proportional to:

$$
S_{1 s t}=\frac{1}{3} \partial_{\nu} \xi_{[1}^{\rho} \partial_{\rho} \xi_{2}^{\sigma} \partial_{\sigma} \xi_{3]}^{\nu}
$$

It has the distinguishing feature of being anti-symmetrized over the indices $1,2,3$. The ambiguity in the Jacobiator will be present in all future computations. In the following, we will prefer to work with brackets which are anti-symmetrized, and which correspond (when relevant) to Courant brackets at lowest order. It is tedious but straightforward to work out the Jacobiators for all other choices of total derivative terms.

\section{B.1.3 A purely left gauge theory algebra}

In this subsection, we want to study a second example of a chiral algebra of currents and charges. The algebra is embedded in the vertex operator algebra of a free chiral scalar field $X$. We can think of $X=X_{L}$ as holomorphic, and we will again denote its left momentum $k_{L}$ by $k$ in this section. We want to compute the (chiral) algebra of the operators:

$$
Q_{L}=\frac{1}{2 \pi i} \oint \xi^{L}(X) \cdot \partial X
$$

However, now we work with the elementary operator product:

$$
X^{\mu}(z) X^{\nu}(w) \sim-\eta^{\mu \nu} \log (z-w) .
$$

After Fourier decomposition, and noting the operator product equality

$$
: e^{i k_{i} \cdot X(z)}:: e^{i k_{j} \cdot X(w)}:=z^{\frac{\alpha^{\prime}}{2} k_{i} \cdot k_{j}}: e^{i k_{i} \cdot X(z)} e^{i k_{j} \cdot X(w)}:
$$

we see that to have a strict vertex operator algebra, we need $k_{i} \cdot k_{j} \in 2 \mathbb{Z}$. In the following, we make the stronger assumption $k_{i} \cdot k_{j}=0$, and we will remark on it when pertinent. Another difference with the algebra of subsection B.1.2 will be that additional terms are generated because $\partial X$ will contract with $\partial X$ and $e^{i k X}$. After a calculation very similar to that of the previous subsection, we find the commutator of these charges:

$$
\left[Q_{L}\left(\xi_{1}^{L}\right), Q_{L}\left(\xi_{2}^{L}\right)\right]=-Q_{L}\left(\left[\xi_{1}^{L}, \xi_{2}^{L}\right]\right)+Q_{L}\left(\omega\left(\xi_{1}^{L}, \xi_{2}^{L}\right)\right)
$$

where we have defined:

$$
\omega_{\mu}\left(\xi_{1}^{L}, \xi_{2}^{L}\right)=-\frac{1}{2}\left(\partial_{\mu} \xi_{1}^{L} \cdot \xi_{2}^{L}-\partial_{\mu} \xi_{2}^{L} \cdot \xi_{1}^{L}\right)+\frac{\lambda_{3}}{2}\left(\partial_{\mu} \partial_{\rho} \xi_{1}^{L \sigma} \partial_{\sigma} \xi_{2}^{L \rho}-\partial_{\mu} \partial_{\rho} \xi_{2}^{L \sigma} \partial_{\sigma} \xi_{1}^{L \rho}\right),
$$

and $\lambda_{3}$ is proportional to $\alpha^{\prime}$. Again, the Jacobi identity will be satisfied by the general theory of vertex operator algebras. If we record the algebra in terms of the parameters of the charges, we can compute the parameter of the Jacobiator to be a total derivative. It is explicitly given by :

$$
\begin{aligned}
J_{L} & =\partial_{\mu} S_{L} \\
S_{L} & =\frac{1}{8}\left(\xi^{[1 \nu} \xi^{2 \rho} K_{\nu \rho}^{3]}+\lambda_{3} \xi^{[1 \nu} K^{2 \rho \sigma} \partial_{\nu} K_{\rho \sigma}^{3]}+\frac{2 \lambda_{3}}{3} K_{\nu}^{\left[1^{\rho}\right.} K_{\rho}^{2^{\sigma}} K_{\sigma}^{3]^{\nu}}\right)
\end{aligned}
$$

where

$$
K_{\mu \nu}=\partial_{\mu} \xi_{\nu}-\partial_{\nu} \xi_{\mu} .
$$

An identical right algebra associated to an anti-holomorphic vertex operator algebra can be constructed. In our final formula, we put the Jacobiator in a form that is easy to match to [20]. 


\section{Bootstrapping a purely left algebra}

In this subsection, we take an alternative approach to finding higher derivative corrections. We analyze a class of extensions of the lowest order algebra and demand that the extension still satisfy the Jacobi identity for charges (or that the Jacobiator of parameters is a total derivative). We set up the problem with the ansatz:

$$
\left[Q_{L}\left(\xi_{1}^{L}\right), Q_{L}\left(\xi_{2}^{L}\right)\right]=-Q_{L}\left(\left[\xi_{1}^{L}, \xi_{2}^{L}\right]\right)+Q_{L}\left(\omega\left(\xi_{1}^{L}, \xi_{2}^{L} ; \lambda_{1}, \lambda_{3}, \epsilon_{1}, \epsilon_{3}\right)\right)
$$

where we define:

$$
\begin{aligned}
\omega_{\mu}\left(\xi_{1}^{L}, \xi_{2}^{L} ; \lambda_{1}, \lambda_{3}, \epsilon_{1}, \epsilon_{3}\right)= & -\frac{\lambda_{1}}{2}\left(\partial_{\mu} \xi_{1}^{L} \cdot \xi_{2}^{L}-\partial_{\mu} \xi_{2}^{L} \cdot \xi_{1}^{L}\right)+\epsilon_{1} \partial_{\mu}\left(\xi_{1}^{L} \cdot \xi_{2}^{L}\right) \\
& -\frac{\lambda_{3}}{2}\left(\partial_{\mu} \partial_{\rho} \xi_{1}^{L \sigma} \partial_{\sigma} \xi_{2}^{L \rho}-\partial_{\mu} \partial_{\rho} \xi_{2}^{L \sigma} \partial_{\sigma} \xi_{1}^{L \rho}\right)+\epsilon_{3} \partial_{\mu}\left(\partial_{\rho} \xi_{1}^{L \sigma} \partial_{\sigma} \xi_{2}^{L \rho}\right) .
\end{aligned}
$$

We want to check the Jacobi identity for generic parameters $\lambda_{1}, \lambda_{3}$ and the impact of the total derivatives which we can add to $\omega_{\mu}$. We can then prove the following results. When $\lambda_{3}=0$, we find that $\lambda_{1}=0$ or $\lambda_{1}=1$ are the only two solutions to the Jacobi identity, independently of the values of $\epsilon_{1}, \epsilon_{3}$. If we have that $\lambda_{3} \neq 0$, then we must have $\lambda_{1}=1$. In other words, we cannot add the $\lambda_{3}$ term without adding the $\lambda_{1}=1$ term. On the dimensionful parameter $\lambda_{3}$ there is no further condition. The anti-symmetric choice of total derivative term $\left(\epsilon_{1}=\epsilon_{3}=0\right)$ matches the bracket in equation (B.17).

\section{B.2 A non-chiral algebra}

In this section, we analyze how the algebras are modified when the left- and right-moving sectors communicate. The general theory of vertex operator algebras, and their tensor products, will now no longer guarantee a total derivative Jacobiator. We can thus expect new algebraic structures. We focus on the example of the algebra of vertex operators associated to diffeomorphism and anti-symmetric gauge parameters.

First of all, we remark that a pedagogical classical derivation of the Courant bracket of local invariances on the string worldsheet is given in [17. Moreover, a clear explanation of the Courant bracket and why it arises from non-commutativity of diffeomorphisms and antisymmetric gauge transformations and that it has enhanced symmetry properties has been reviewed in [18, where further original references can be found.

Here we will be interested in higher derivative corrections to the algebra of (generalized) diffeomorphism vertex operators. To simplify our life, we will consider a model of chiral bosons, both left and right, and ignore contact terms. Thus, we consider the following algebras of chiral fields:

$$
\begin{aligned}
& X_{L}(z) X_{L}(w) \sim-\log (z-w) \\
& X_{R}(\bar{z}) X_{R}(\bar{w}) \sim-\log (\bar{z}-\bar{w}) .
\end{aligned}
$$

To mix the left and the right algebras in this model, we introduce the following charges:

$$
\begin{aligned}
Q_{L} & =\oint d z \xi_{L}\left(X_{L}(z), X_{R}(\bar{z})\right) \partial X_{L}(z) \\
Q_{R} & =\oint d \bar{z} \xi_{R}\left(X_{R}(\bar{z}), X_{L}(z)\right) \bar{\partial} X_{R}(\bar{z}) .
\end{aligned}
$$


We leave the basic operator product expansions unchanged. The resulting algebra is:

$$
\begin{aligned}
{\left[Q_{L}\left(\xi_{1}^{L}\right), Q_{L}\left(\xi_{2}^{L}\right)\right]=} & Q_{L}\left(\left[\xi_{1}^{L}, \xi_{2}^{L}\right]\right)+\frac{1}{2} Q_{L}\left(\partial_{\mu}^{L} \xi_{1}^{\rho} \xi_{2 \rho}-\partial_{\mu}^{L} \xi_{2}^{\rho} \xi_{1 \rho}\right)-\frac{1}{2} Q_{R}\left(\partial_{\mu}^{R} \xi_{1}^{\rho} \xi_{2 \rho}-\partial_{\mu}^{R} \xi_{2}^{\rho} \xi_{1 \rho}\right) \\
& +\frac{\alpha^{\prime}}{4} Q_{L}\left(\partial_{\mu}^{L} \partial_{\sigma}^{L} \xi_{1}^{\rho} \partial_{\rho}^{L} \xi_{2}^{\sigma}-\partial_{\mu}^{L} \partial_{\sigma}^{L} \xi_{2}^{\rho} \partial_{\rho}^{L} \xi_{1}^{\sigma}\right)-\frac{\alpha^{\prime}}{4} Q_{R}\left(\partial_{\mu}^{R} \partial_{\sigma}^{L} \xi_{1}^{\rho} \partial_{\rho}^{L} \xi_{2}^{\sigma}-\partial_{\mu}^{R} \partial_{\sigma}^{L} \xi_{2}^{\rho} \partial_{\rho}^{L} \xi_{1}^{\sigma}\right),
\end{aligned}
$$

and an identical right algebra (with left and right interchanged). The left-right algebra is

$$
\begin{aligned}
{\left[Q_{L}\left(\xi_{1}^{L}\right), Q_{R}\left(\xi_{2}^{R}\right)\right]=} & -Q_{L}\left(\xi^{R 2 \rho} \partial_{\rho}^{R} \xi^{L 1}\right)+Q_{R}\left(\xi^{L 1 \rho} \partial_{\rho}^{L} \xi^{R 2}\right) \\
& +\frac{\alpha^{\prime}}{4} Q_{L}\left(\partial_{\mu}^{L} \partial_{\rho}^{R} \xi^{L 1 \nu} \partial_{\nu}^{L} \xi^{R 2 \rho}-\partial_{\mu}^{L} \partial_{\rho}^{L} \xi^{R 2 \nu} \partial_{\nu}^{R} \xi^{L 1 \rho}\right) \\
& +\frac{\alpha^{\prime}}{4} Q_{R}\left(\partial_{\mu}^{R} \partial_{\rho}^{R} \xi^{L 1 \nu} \partial_{\nu}^{L} \xi^{R 2 \rho}-\partial_{\mu}^{R} \partial_{\rho}^{L} \xi^{R 2 \nu} \partial_{\nu}^{R} \xi^{L 1 \rho}\right)
\end{aligned}
$$

This algebra is useful in doubled geometry applications. When we identify derivatives with respect to left and right coordinates, we find the algebra:

$$
\begin{aligned}
{\left[Q_{L}\left(\xi_{1}^{L}\right), Q_{L}\left(\xi_{2}^{L}\right)\right]=} & Q_{L}\left(\left[\xi_{1}^{L}, \xi_{2}^{L}\right]\right)+\frac{1}{2} Q_{L}\left(\partial_{\mu} \xi_{1}^{\rho} \xi_{2 \rho}-\partial_{\mu} \xi_{2}^{\rho} \xi_{1 \rho}\right)-\frac{1}{2} Q_{R}\left(\partial_{\mu} \xi_{1}^{\rho} \xi_{2 \rho}-\partial_{\mu} \xi_{2}^{\rho} \xi_{1 \rho}\right) \\
& +\frac{\alpha^{\prime}}{4} Q_{L}\left(\partial_{\mu} \partial_{\sigma} \xi_{1}^{\rho} \partial_{\rho} \xi_{2}^{\sigma}-\partial_{\mu} \partial_{\sigma} \xi_{2}^{\rho} \partial_{\rho} \xi_{1}^{\sigma}\right)-\frac{\alpha^{\prime}}{4} Q_{R}\left(\partial_{\mu} \partial_{\sigma} \xi_{1}^{\rho} \partial_{\rho} \xi_{2}^{\sigma}-\partial_{\mu} \partial_{\sigma} \xi_{2}^{\rho} \partial_{\rho} \xi_{1}^{\sigma}\right) .
\end{aligned}
$$

We also find the mixing:

$$
\begin{aligned}
{\left[Q_{L}\left(\xi_{1}^{L}\right), Q_{R}\left(\xi_{2}^{R}\right)\right]=} & -Q_{L}\left(\xi^{R 2 \rho} \partial_{\rho} \xi^{L 1}\right)+Q_{R}\left(\xi^{L 1 \rho} \partial_{\rho} \xi^{R 2}\right) \\
& +\frac{\alpha^{\prime}}{4} Q_{L}\left(\partial_{\mu} \partial_{\rho} \xi^{L 1 \nu} \partial_{\nu} \xi^{R 2 \rho}-\partial_{\mu} \partial_{\rho} \xi^{R 2 \nu} \partial_{\nu} \xi^{L 1 \rho}\right) \\
& +\frac{\alpha^{\prime}}{4} Q_{R}\left(\partial_{\mu} \partial_{\rho} \xi^{L 1 \nu} \partial_{\nu} \xi^{R 2 \rho}-\partial_{\mu} \partial_{\rho} \xi^{R 2 \nu} \partial_{\nu} \xi^{L 1 \rho}\right) .
\end{aligned}
$$

This is the $\alpha^{\prime}$ corrected algebra of generalized anti-symmetric gauge transformation and diffeomorphism vertex operators in a left-right separated basis. To go to a standard basis, we use the linear map:

$$
\begin{array}{rr}
D(\xi)=\frac{1}{2}\left(Q_{L}(\xi)+Q_{R}(\xi)\right) & A(\tilde{\xi})=\frac{1}{2}\left(Q_{L}(\tilde{\xi})-Q_{R}(\tilde{\xi})\right) \\
Q_{L}\left(\xi_{L}\right)=D\left(\xi_{L}\right)+A\left(\xi_{L}\right) & Q_{R}\left(\xi_{R}\right)=D\left(\xi_{R}\right)-A\left(\xi_{R}\right),
\end{array}
$$

and find:

$$
\begin{aligned}
& {\left[D\left(\xi^{1}\right), D\left(\xi^{2}\right)\right]=D\left(\left[\xi^{1}, \xi^{2}\right]^{\mu}\right)+\frac{1}{2} D\left(\partial^{\mu} \partial_{\rho} \xi^{1 \sigma} \partial_{\sigma} \xi^{2 \rho}-\partial^{\mu} \partial_{\rho} \xi^{2 \sigma} \partial_{\sigma} \xi^{1 \rho}\right)} \\
& {\left[A\left(\tilde{\xi}^{1}\right), A\left(\tilde{\xi}^{2}\right)\right]=-\frac{1}{2} D\left(\partial^{\mu} \partial_{\rho} \tilde{\xi}^{1 \sigma} \partial_{\sigma} \tilde{\xi}^{2 \rho}-\partial^{\mu} \partial_{\rho} \tilde{\xi}^{2 \sigma} \partial_{\sigma} \tilde{\xi}^{1 \rho}\right)} \\
& {\left[D\left(\xi^{1}\right), A\left(\tilde{\xi}^{2}\right)\right]=A\left(\mathcal{L}_{\xi^{1}} \tilde{\xi}^{2}-\frac{1}{2} d\left(\xi^{1} \cdot \tilde{\xi}^{2}\right)+\frac{1}{2}\left(\partial^{\mu} \partial_{\rho} \xi^{1 \sigma} \partial_{\sigma} \tilde{\xi}^{2 \rho}-\partial^{\mu} \partial_{\rho} \tilde{\xi}^{2 \sigma} \partial_{\sigma} \xi^{1 \rho}\right)\right) .}
\end{aligned}
$$


The Jacobiators in this basis are:

$$
\begin{aligned}
{\left[D\left(\xi^{1}\right),\left[D\left(\xi^{2}\right), D\left(\xi^{3}\right)\right]\right]+\text { cycl. perm. }=} & D\left[-\frac{1}{6} \partial_{\mu}\left(\partial_{\alpha} \xi^{[1 \beta} \partial_{\beta} \xi^{2 \gamma} \partial_{\gamma} \xi^{3] \alpha}\right)\right. \\
& \left.-\frac{1}{2}\left(\partial_{\mu} \xi^{1 \rho} \partial_{\rho} \partial_{\alpha} \xi^{[2 \beta} \partial_{\beta} \xi^{3] \alpha}+\text { cycl. perm. }\right)\right] \\
= & D\left[-\frac{1}{12} \partial_{\mu}\left(K_{\alpha}^{[1 \beta} K_{\beta}^{2 \gamma} K_{\gamma}^{3]^{\alpha}}\right)-\frac{1}{4} \partial_{\mu} \xi^{[1 \nu} K^{2 \rho \sigma} \partial_{\nu} K_{\rho \sigma}^{3]}\right]
\end{aligned}
$$

$\left[A\left(\tilde{\xi}^{1}\right),\left[A\left(\tilde{\xi}^{2}\right), A\left(\tilde{\xi}^{3}\right)\right]\right]+$ cycl. perm. $=0$

$$
\begin{aligned}
{\left[A\left(\tilde{\xi}^{1}\right),\left[A\left(\tilde{\xi}^{2}\right), D\left(\xi^{3}\right)\right]\right]+\text { cycl. perm. }=} & D\left[\frac{1}{2} \partial_{\mu}\left(\partial_{\alpha} \tilde{\xi}^{[1 \beta} \partial_{\beta} \tilde{\xi}^{2] \gamma} \partial_{\gamma} \xi^{3 \alpha}\right)\right. \\
& \left.-\frac{1}{2} \partial_{\alpha} \tilde{\xi}^{[1 \beta} \partial_{\gamma} \partial_{\beta} \tilde{\xi}^{2] \alpha} \partial_{\mu} \xi^{3 \gamma}-\partial_{\alpha} \tilde{\xi}^{[1 \beta} \partial_{\mu} \partial_{\beta} \tilde{\xi}^{2] \gamma} \partial_{\gamma} \xi^{3 \alpha}\right] \\
= & D\left[\frac{1}{12} \partial_{\mu}\left(\tilde{K}_{\alpha}^{[1 \beta} \tilde{K}_{\beta}^{2 \gamma} K_{\gamma}^{3]^{\alpha}}\right)\right. \\
& \left.-\frac{1}{4} \tilde{K}_{\alpha}^{[1 \beta} \partial_{\gamma} \tilde{K}_{\beta}^{2] \alpha} \partial_{\mu} \xi^{3 \gamma}-\tilde{K}_{\alpha}^{[1 \beta} \partial_{\mu} \tilde{K}_{\beta}^{2] \gamma} \partial_{\gamma} \xi^{3 \alpha}\right]
\end{aligned}
$$

$$
\begin{aligned}
{\left[D\left(\xi^{1}\right),\left[D\left(\xi^{2}\right), A\left(\tilde{\xi}^{3}\right)\right]\right]+\text { cycl. perm. }=} & A\left[\frac{1}{4} \partial_{\mu}\left(\xi^{[1 \alpha} \xi_{\nu}^{2]} \partial_{\alpha} \tilde{\xi}^{3 \nu}+\partial_{\alpha} \xi_{\nu}^{[1} \xi^{2] \alpha} \tilde{\xi}^{3 \nu}\right)\right. \\
& -\frac{1}{4} \partial_{\mu}\left(\xi^{1 \nu} \partial_{\nu} \partial_{\alpha} \xi^{[2 \beta} \partial_{\beta} \tilde{\xi}^{3] \alpha}+\text { cycl. perm. }\right) \\
& \left.-\frac{1}{2}\left(2 \partial_{\alpha} \xi^{[1 \beta} \partial_{\mu} \partial_{\beta} \xi^{2] \gamma} \partial_{\gamma} \tilde{\xi}^{3 \alpha}-\partial_{\nu} \partial_{\alpha} \xi^{[1 \beta} \partial_{\beta} \xi^{2] \alpha} \partial_{\mu} \tilde{\xi}^{3 \nu}\right)\right] \\
= & A\left[\frac{1}{4} \partial_{\mu}\left(\xi^{[1 \alpha} \xi_{\nu}^{2]} \partial_{\alpha} \tilde{\xi}^{3 \nu}+\partial_{\alpha} \xi_{\nu}^{[1} \xi^{2] \alpha} \tilde{\xi}^{3 \nu}-\frac{1}{2} \xi^{[1 \nu} K^{2 \rho \sigma} \partial_{\nu} \tilde{K}_{\rho \sigma}^{3]}\right)\right] \\
& \left.-\left(K_{\alpha}^{[1 \beta} \partial_{\mu} K_{\beta}^{2]^{\gamma}} \partial_{\gamma} \tilde{\xi}^{3 \alpha}-\frac{1}{4} \partial_{\nu} K_{\alpha}^{\left[1^{\beta}\right.} K_{\beta}^{2]^{\alpha}} \partial_{\mu} \tilde{\xi}^{3 \nu}\right)\right]
\end{aligned}
$$

We use the anti-symmetrization convention which assigns unit weight to each term in the permutation sum. We also have the useful identities (under the assumption that contracted momenta are neglected):

$$
\begin{aligned}
K_{\alpha}^{1^{\beta}} K_{\beta}^{2 \gamma} K_{\gamma}^{3^{\alpha}} & =\partial_{\alpha} \xi^{1 \beta} \partial_{\beta} \xi^{2 \gamma} \partial_{\gamma} \xi^{3 \alpha}-\partial_{\alpha} \xi^{1 \beta} \partial_{\beta} \xi^{3 \gamma} \partial_{\gamma} \xi^{2 \alpha} \\
\xi^{[1 \nu} K^{2 \rho \sigma} \partial_{\nu} K_{\rho \sigma}^{3]} & =-2 \xi^{1 \nu}\left(\partial^{\rho} \xi^{2 \sigma} \partial_{\nu} \partial_{\sigma} \xi_{\rho}^{3}-\partial^{\rho} \xi^{3 \sigma} \partial_{\nu} \partial_{\sigma} \xi_{\rho}^{2}\right)+\text { cycl. perm. } \\
K_{\alpha}^{1 \beta} K_{\beta}^{2 \alpha} & =2 \partial_{\alpha} \xi^{1 \beta} \partial_{\beta} \xi^{2 \alpha}
\end{aligned}
$$

In the total derivative term, the Jacobiator of diffeomorphisms is anti-symmetric and linear in the three parameters $\xi_{1,2,3}$. Moreover, the extra terms arise from a derivative with an external leg acting on one of the three parameters. As such, it may allow for an embedding into 
a strongly homotopy Lie algebra. This may be the case for the full algebra of diffeomorphisms and anti-symmetric gauge transformations. We leave the closer study of the algebra, as well as the broader question to how this algebraic structure is encoded in the tensor product of vertex operator algebras for future research. (See e.g. [27] for some inspiration.)

\section{References}

[1] H. Bondi, M. G. J. van der Burg and A. W. K. Metzner, "Gravitational waves in general relativity. 7. Waves from axisymmetric isolated systems," Proc. Roy. Soc. Lond. A 269 (1962) 21.

[2] R. K. Sachs, "Gravitational waves in general relativity. 8. Waves in asymptotically flat space-times," Proc. Roy. Soc. Lond. A 270 (1962) 103.

[3] R. Sachs, "Asymptotic symmetries in gravitational theory," Phys. Rev. 128 (1962) 2851.

[4] R. Penrose, "Asymptotic properties of fields and space-times," Phys. Rev. Lett. 10 (1963) 66.

[5] E. T. Newman and R. Penrose, "Note on the Bondi-Metzner-Sachs group," J. Math. Phys. 7 (1966) 863.

[6] A. Ashtekar, J. Bicak and B. G. Schmidt, "Asymptotic structure of symmetry reduced general relativity," Phys. Rev. D 55 (1997) 669 gr-qc/9608042 AND OTHERS.

[7] G. Barnich and G. Compere, "Classical central extension for asymptotic symmetries at null infinity in three spacetime dimensions," Class. Quant. Grav. 24 (2007) F15 gr-qc/0610130].

[8] G. Barnich and C. Troessaert, "Aspects of the BMS/CFT correspondence," JHEP 1005 (2010) 062 arXiv:1001.1541 [hep-th]].

[9] A. Bagchi, S. Detournay, R. Fareghbal and J. Simon, "Holography of 3d Flat Cosmological Horizons," Phys. Rev. Lett. 110 (2013) 141302 [arXiv:1208.4372 [hep-th]].

[10] J. D. Brown and M. Henneaux, "Central Charges in the Canonical Realization of Asymptotic Symmetries: An Example from Three-Dimensional Gravity," Commun. Math. Phys. 104 (1986) 207.

[11] G. Barnich, A. Gomberoff and H. A. Gonzalez, "The Flat limit of three dimensional asymptotically anti-de Sitter spacetimes," Phys. Rev. D 86 (2012) 024020 arXiv:1204.3288 [grqc]].

[12] J. Troost, "The $A d S_{3}$ boundary energy momentum tensor, exact in the string length over the curvature radius," Phys. Lett. B 689 (2010) 201 [arXiv:1006.4223 [hep-th]].

[13] J. Troost, "The $A d S_{3}$ central charge in string theory," Phys. Lett. B 705 (2011) 260 arXiv:1109.1923 [hep-th]].

[14] A. Giveon, D. Kutasov and N. Seiberg, "Comments on string theory on AdS(3)," Adv. Theor. Math. Phys. 2 (1998) 733 [hep-th/9806194].

[15] D. Kutasov and N. Seiberg, "More comments on string theory on AdS(3)," JHEP 9904 (1999) 008 hep-th/9903219. 
[16] S. K. Ashok, R. Benichou and J. Troost, "Conformal Current Algebra in Two Dimensions," JHEP 0906 (2009) 017 arXiv:0903.4277 [hep-th]].

[17] A. Alekseev and T. Strobl, "Current algebras and differential geometry," JHEP 0503 (2005) 035 hep-th/0410183.

[18] B. Zwiebach, "Double Field Theory, T-Duality, and Courant Brackets," Lect. Notes Phys. 851 (2012) 265 [arXiv:1109.1782 [hep-th]].

[19] A. S. Losev, A. Marshakov and A. M. Zeitlin, "On first order formalism in string theory," Phys. Lett. B 633 (2006) 375 hep-th/0510065.

[20] O. Hohm, W. Siegel and B. Zwiebach, "Doubled $\alpha$ '-Geometry," arXiv:1306.2970 [hep-th].

[21] A. Strominger, Talk at Strings 2013, 26 June 2013.

[22] J. Polchinski, "String theory. Vol. 1: An introduction to the bosonic string," Cambridge, UK: Univ. Pr. (1998) 402 p.

[23] V. Kac, "Vertex algebras for beginners," Providence, USA: AMS (1996) 141 p. (University lectures series. 10)

[24] D. Pinzon, "Vertex operator algebras and strongly homotopy Lie algebras," PhD. Thesis, Kentucky.

[25] B. Zwiebach, "Closed string field theory: Quantum action and the B-V master equation," Nucl. Phys. B 390 (1993) 33 [hep-th/9206084].

[26] J. Stasheff, "Closed string field theory, strong homotopy Lie algebras and the operad actions of moduli space," In *Penner, R. (ed.): Perspectives in mathematical physics* 265-288. hep-th/9304061.

[27] A. M. Zeitlin, "Beta-gamma systems and the deformations of the BRST operator," J. Phys. A 42 (2009) 355401 [arXiv:0904.2234 [hep-th]]. 